\title{
Dispersive Properties of Self-Complementary Log-Periodic Antennas in Pulsed THz Systems
}

\author{
A.D.J. Fernandez Olvera*, U. Nandi*, J. Norman, ${ }^{\dagger}$, A. C. Gossard ${ }^{\dagger}$, H. Roskos ${ }^{\ddagger}$ and S. Preu* \\ ${ }^{*}$ Dept. of Electrical Engineering and Information Technology, TU Darmstadt, Germany \\ $\dagger$ Materials Department, University of California, Santa Barbara, USA \\ $\ddagger$ Physics Dept., Johann Wolfgang Goethe University Frankfurt, Germany
}

\begin{abstract}
We present a simple analytical model for the dispersion of a pulse radiated by a self-complementary logarithmicperiodic antenna. The model predicts the variation in the spectral phase, and explains the underlying mechanism behind it. The variation in the spectral phase predicted by the model is compared with the spectral phase measured with a Terahertz (THz) Time Domain System (TDS) System that uses the same type of antenna.
\end{abstract}

\section{INTRODUCTION}

Self-Complementary logarithmic-periodic antennas are used extensively in continuous wave $\mathrm{THz}$ systems due to their large bandwidth and constant radiation resistance. However, they are barely used in pulsed systems since they show a dispersive behavior, distorting the pulse. We present a simple model for this behavior based on the varying delay that each frequency component experiences before getting radiated. The results are then compared to the measurements obtained with logarithmic-periodic antenna in a THz TDS. In principle, the results presented here are valid for any type of logarithmicperiodic antenna.

\section{DISPERSION MODEL}

In a logarithmic-periodic self-complementary antenna, like the one shown in Fig. 1, each frequency component within the operational range of the antenna is radiated by its corresponding resonant arm. The lower frequencies of the operational range are radiated by the outer arms of the antenna. Hence, the current has to travel a longer distance before getting radiated as compared to high frequencies. The temporal delay of the lower frequencies provokes dispersion of the pulse, resulting in a chirped pulse with low frequencies being emitted at a later time. In the frequency domain, the temporal delay results in a variation of the spectral phase which will be calculated in the following and compared to experimental results.

Our model is based on the following assumptions:

- The dispersion is only caused by the increasing distance that the lower frequency components have to travel before getting radiated.

- The resultant frequency-dependent delay, $\tau(f)$, is smooth over the bandwidth of interest, so that the spectral phase can be calculated using [1]:

$$
\tau(f)=-\frac{1}{2 \pi} \frac{d \phi}{d f}
$$

where $\phi$ is the phase, and $f$ is the frequency.

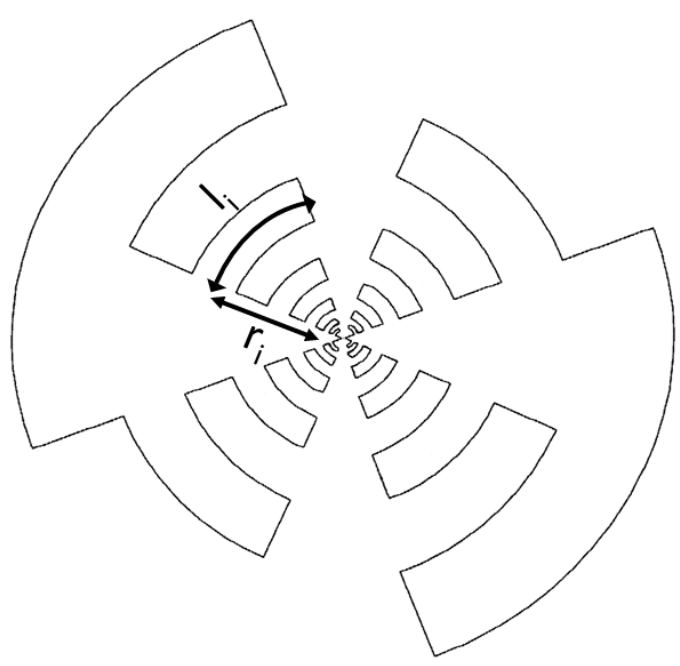

Fig. 1. Schematic representation of the self-complementary logarithmicperiodic antenna used in the THz TDS setup.

In the logarithmic-periodic antenna used [2], illustrated in Fig. 1, each radiated frequency component $f_{i}$ is related to the (average) radius of the respective resonant arm, $r_{i}$, by

$$
f_{i}=\frac{2 c_{0}}{\pi n_{e f f} r_{i}},
$$

where $c_{0}$ is the speed of light, $n_{\text {eff }}$ the effective refractive index. This relationship results from the fact that the length of each resonant arm, $l_{i}$, equals one eighth of the circumference defined by $r_{i}$, and that each arm is resonant when its center length equals half a wavelength, $\lambda_{i} / 2$.

Similar relations can be found for any other types of logarithmic-periodic antenna like spirals or infinite bow tie antennas, generally resulting in

$$
f l=\text { const }
$$

where $l$ is the distance travelled by frequency component $f$. This simple relation solely results from the periodic nature of the antenna; for an $n$ times higher frequency the resonant part of the antenna is a factor $n$ closer to the center.

Since $\tau(f) \sim l$ we can re-express Eq. 3 in terms of the time delay 


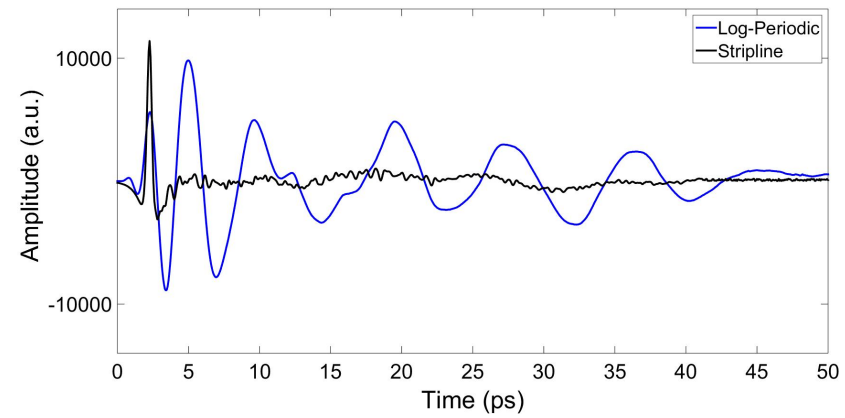

Fig. 2. Comparison between the pulse emitted by strip-line and logarithmicperiodic antenna in the time domain.

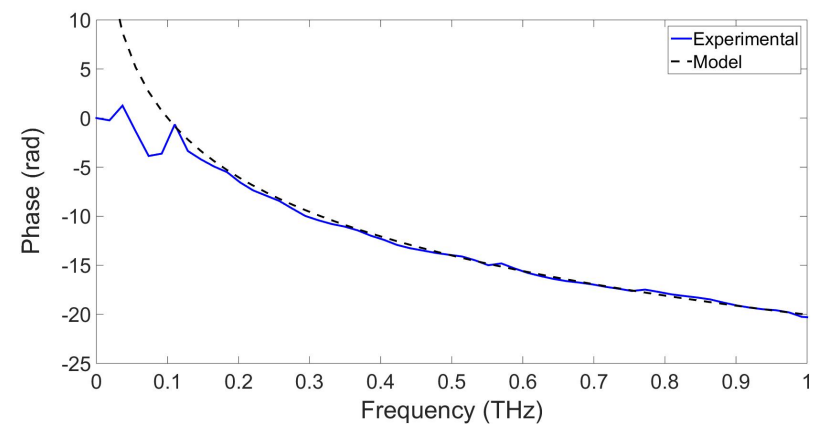

Fig. 3. Comparison between the spectral phase of the pulse emitted by the logarithmic-periodic antenna and the theoretical spectral phase from Eq. 3 .

$$
\tau(f) \sim \frac{1}{f},
$$

Substituting Eq. 4 in Eq. 1 and solving for $\phi(f)$ yields

$$
\phi(f)=-\phi_{0} \ln \left(\frac{f}{f_{0}}\right),
$$

where $f_{0}$ is a frequency normalization constant that depends on the operational range of the antenna, and $\phi_{0}$ is a proportionality constant that depends on the speed of light in the medium.

It is worthwhile to mention that other authors have attributed the dispersion to a logarithmic variation of the velocity of the wave as a function of the frequency [4]. Such variation is caused by the non-uniform distribution of the resonant arms. This variation gets more pronounced as the frequency decreases, since the wave starts seeing the effect of more of these arms before it actually gets radiated [5]. Using this more complex approach one gets the same result as in Eq. 5 .

\section{COMPARISON WITH EXPERIMENTAL RESULTS}

To prove the validity of the theoretical considerations, the spectral phase from Eq. 4 is compared with the spectral phase measured by a THz TDS system. The TDS system consists of two ErAs:In(Al)GaAs photoconductive antennas [3] excited by a 90 fs laser pulse with a center frequency of $1550 \mathrm{~nm}$.
In order to discard any external sources of dispersion in our comparison, the response of the system is tested with two non-dispersive antennas first. The antennas selected for this purpose are two strip-line antennas with a photoconductive active area of $25 \mu \mathrm{m} \times 25 \mu \mathrm{m}$; one of them acting as a transmitter and the other one acting as a receiver. The response of the system in the time domain is indicated by the dotted line in Fig. 2. The pulse is not chirped, hence, we can conclude that the system is well aligned and that there are no other sources of dispersion.

For the comparison with Eq. 4, the transmitting stripline antenna is replaced by the logarithmic-periodic antenna illustrated in Fig 1, with a photoconductive active area of 10 $\mu \mathrm{m} \times 10 \mu \mathrm{m}$. The response of the system in the time domain is shown by the solid line in Fig. 2. The pulse is highly chirped due to the dispersion introduced by the antenna as expected from the theoretical considerations. The spectral phase of the pulse is obtained by taking the Fourier Transform, and then extracting the phase. Fig. 3 compares the experimentally obtained phase to the one predicted by Eq. 4 using $f_{0}=100$ $\mathrm{GHz}$ and $\phi_{0}=8.7 \mathrm{rad}$. The two curves show excellent agreement. The discrepancy for frequencies below $100 \mathrm{GHz}$ has two origins: firstly, the wave experiences the finite size of the antenna, secondly, the finite time window causes artifacts for low frequencies, e.g. the known divergence at $f \rightarrow 0$.

\section{CONCLUSiOn AND OUTLOOK}

We have shown that the dispersion in a logarithmic-periodic antenna is caused by the logarithmic change of the spectral phase of the current, corresponding to an increasing time delay for lower frequencies. The separation of the frequency components in time may allow for selecting specific frequency intervals by time-domain gating around the delay time of the wanted frequency range.

\section{ACKNOWLEDGMENT}

The authors acknowledge funding by the Deutsche Forschungsgemeinschaft Project PR1413/3-1 (REPHCON) and European Union's Horizon 2020 Research and Innovation Program 675683 (CELTA).

\section{REFERENCES}

[1] J. O. Smith, Frequency Response Analysis, in Introduction to Digital Filters with Audio Applications, W3K Publishing, 2007, ch. 7.

[2] Y. Huo, G. W. Taylor, and R. Bansal, "Planar Log-Periodic Antennas on Extended Hemispherical Silicon Lenses for Millimiter/Submillimeter Wave Detection Applications", International Journal of Infrared and Millimeter Waves, pp. 819-839 (2002).

[3] S. Preu, et al. "1550 nm ErAs: In(A1)GaAs large area photoconductive emitters," Appl. Phys. Lett., vol 101,p. 101105 (2012).

[4] C. M. Knop, On Transient Radiation from a Log-Peroidic Dipole Array, IEEE Transactions on Antennas and Propagation, pp. 807-808, November 1970.

[5] E.C. Jordan, et al., Developments in broadband antennas, IEEE Spectrum, pp. 58-71, 1964. 